\title{
Soil microbes and oxygen influence the changes in bacterial community composition during swine carcass decomposition
}

\author{
Michelle Miguel \\ Sunchon National University \\ Seon Ho Kim \\ Sunchon National University \\ Sang Suk Lee \\ Sunchon National University \\ Yong II Cho ( $\square$ ycho@scnu.ac.kr) \\ Sunchon National University
}

\section{Research Article}

Keywords: 16S rRNA, bacterial diversity, swine, decomposition, miseq

Posted Date: February 25th, 2021

DOI: https://doi.org/10.21203/rs.3.rs-211559/v1

License: @ (i) This work is licensed under a Creative Commons Attribution 4.0 International License. Read Full License 


\section{Abstract \\ Background}

Carcass decomposition is influenced by various factors such as temperature, humidity, microorganisms, invertebrates, and scavengers. Soil microbes play a significant role in the decomposition process. In this study, we investigated the changes in the bacterial community during carcass decomposition in soil with an intact microbial community and soil which was sterilized decomposed with and without oxygen access using 16 s rRNA metagenomic sequencing.

\section{Results}

Based on the 16S rRNA metagenomic sequencing, a total of 988 operational taxonomic units (OTUs) representing 16 phyla and 533 genera were detected. The bacterial diversity varied across the based on the alpha diversity indices. The bacterial composition in the unsterilized soil - aerobic condition (U_A) and unsterilized soil - anaerobic condition (U_An) set-ups have higher alpha diversity than the other burial set-ups. Beta diversity analysis revealed a close association in the samples according to the burial type and decomposition day. Firmicutes was the dominant phylum across all samples regardless of the burial type and decomposition day. The bacterial community composition changed throughout the decomposition process in all burial set-up. Meanwhile, the genus Bacillus dominated the bacterial community towards the end of decomposition period.

\section{Conclusions}

Our results showed that bacterial community composition changed during carcass decomposition and was affected by the soil and oxygen access, with microorganisms belonging to phylum Firmicutes dominating the community.

\section{Background}

Decomposition is an important natural phenomenon which is influenced by several factors, including temperature, humidity and the activities of microorganisms, invertebrates and scavengers [1]. Various disposal methods have been used for deceased livestock carcasses such as soil burial, burning, incineration, rendering, anaerobic digestion, alkaline hydrolysis and composting have been used for the disposal of livestock carcasses [2, 3]. Among them, soil burial is the most common method used for the disposal of a large number of diseased animal carcasses [3].

Microbial communities are abundant in the environment. Several studies have reported the role of endogenous soil microbial community on carcass decomposition. The decomposition of buried carcasses mainly relies on the [4] microbial production of extracellular proteolytic enzymes that breakup 
polymers of organic matter into oligomeric and monomeric molecules [3]. Soil microbial proteases synthesized in response to the supply of animal organic matter were able to degrade prion protein in vitro [5].

The deposition of animal carcasses and their decomposition can influence the soil microbial communities. This causes great impact in the soil microbiome as the carcass influences both biotic and abiotic factors [6]. In addition, a succession within the microbial community will occur after the introduction of a carcass, hence, changing abundances in accordance with nutrients being released during carcass decomposition [6, 7]. In addition to soil microbes, carcasses also possess a diverse microbiome which is introduced into the soil as decomposition progresses. During the decomposition process, it causes significant and sequential changes in the bacterial communities within the soil and these changes can be correlated with various stages of decomposition.

The microbial communities plays an important role in maintaining soil quality due to their involvement in organic matter dynamics, nutrient cycling and decomposition [8]. However, metabolites from decomposing carcasses can affect the land and surrounding environment [9]. Therefore, it is important to investigate the composition of the bacterial community after soil burial. In our present study, swine carcasses were decomposed on either (i) soil with an intact microbial community (unsterilized soil) or (ii) soil that was sterilized, and each incubated under aerobic (with oxygen) and anaerobic (without oxygen) condition. Metagenomic sequencing of samples obtained through the carcass decomposition was conducted to investigate the bacterial community structure. Specifically, the changes in the bacterial community composition in each experimental set-up was determined.

\section{Results}

\section{Swine carcass decomposition}

Carcass decomposition was conducted in unsterilized and sterilized soil under aerobic and anaerobic conditions. Moisture percentage in the swine carcass was observed for 60 days. Moisture content ranged between $55.75 \%$ to $66.50 \%$ and was relatively constant throughout the experiment, except between days 5 to 10 (Fig. 1a). Significant differences in moisture content between the set-ups were observed at 30 and 60 days of incubation $(p<0.05)$. At 60 days of incubation, higher moisture $(p<0.05)$ was observed in the unsterilized soil-anaerobic burial set-up. In terms of $\mathrm{pH}$, the value ranged from 6.10 to 8.16 . The $\mathrm{pH}$ increased as decomposition progressed and remained relatively constant between days 30 to 60 (Fig. 1b). At the end of day 60 of decomposition, higher pH 8.15 and $8.16(p<0.05)$, respectively, was observed in both the sterilized and unsterilized soil-aerobic condition burial set-ups.

\section{Diversity of bacterial communities}

The changes in the bacterial community during decomposition of swine carcasses over a 60-day period were investigated using Illumina metagenomic sequencing technology. The boxplot representation of alpha diversity indices is shown in Fig. 2. The boxplot of observed OTUs from the samples showed that 
OTUs in unsterilized soil - anaerobic condition (U_An) was higher compared to the other burial set-ups (Fig. 2a). Chao1 which represents the OTU abundance in the samples showed that unsterilized soil anaerobic condition (U_An) was the highest followed by unsterilized soil - aerobic condition (U_A) (Fig. $2 b)$. Shannon index which reflects the diversity of the OTUs in the samples showed that unsterilized soil anaerobic condition (U_An) is the most diverse among the burial set-up and sterilized soil - aerobic condition (S_A) being the least (Fig. 2C). This could indicate that bacterial communities changed at each period and were consisted of various bacterial species. Overall, our results showed that the bacterial composition of unsterilized soil - anaerobic condition (U_An) and unsterilized soil - aerobic condition (U_A) had higher alpha diversity than the other burial set-ups, although no significant difference was observed.

\section{Changes in the bacterial community composition}

Bacterial community composition of each sample was analyzed and compared at the phylum, genus and species levels (Fig. 3-5). A total of 988 OTUs were detected in all samples. Sixteen phyla and 533 genera were identified from the samples in the burial set-ups throughout the decomposition period. Overall, the majority of the sequences belonged to Firmicutes (84\%) (Fig. 3a). At phylum level (Fig. 3b), Firmicutes in the set-up with unsterilized soil both incubated under aerobic and anaerobic condition increased from day 0 to 5 of decomposition. Meanwhile, Firmicutes decreased in abundance from day 0 to 5 in the set-up with sterilized soil both incubated under aerobic and anaerobic condition. In terms of genus level (Fig. 4), the bacterial community composition varied at each sampling day in all burial set-up. During days 0 to 30 , the bacterial community in the samples were dominated by Lactobacillus, Escherichia, and Clostridium. Meanwhile, at days 30 and 60, the samples were dominated by Bacillus. In terms of species level (Fig. 5), the bacterial communities varied at each sampling period. During days 5 and 10, there was a high abundance of Escherichia fergusonii. The dominant bacterial species towards the end of decomposition belongs to Bacillus. Meanwhile, Bacillus haynesii was dominant at day 60 in all burial setups.

The differentially abundant bacterial orders across different burial groups for each sampling period was presented as hierarchical clustering heatmap in Fig. 6. The normalized data presented shows the clustering based on the similarity of relative abundance between representative orders of OTUs (row) and burial groups for each sampling period (column). Lactobacillales was mostly found at day 0 and 5 of decomposition in all groups and was detected in high abundance at day 5 in sterilized soil - aerobic condition (S_A) group. The order Clostridiales was more dominant in U_An at day 5 than in other groups. Meanwhile, Bacillales was mostly present at days 30 and 60 of decomposition in all burial groups. Pseudomonadales has higher abundance unsterilized soil - anaerobic condition (U_An) and sterilized soil - aerobic condition (S_A) group at day 10 of decomposition, while Corynebacteriales was more abundant in sterilized soil - aerobic condition (S_A) at day 10 than in other groups.

The comparison of the bacterial communities by non-metric multidimensional scaling (NMDS) is presented in Fig. 7. Beta diversity did not vary significantly between the different burial groups at different 
time points. Beta diversity analysis revealed a close association between the burial type and sampling period.

\section{Core microbiome}

A Venn diagram was used to compare the similarities and differences between the bacterial communities in the different burial set-ups during carcass decomposition (Fig. 8). The U_A, S_A, U_An, and S_An communities had 308 observed species in common which corresponds to $31.17 \%$ of the total observed species. Meanwhile a total of 400 (40.49\%) were shared by 2 or 3 samples, and $280(28.34 \%)$ of observed species were specific and distributed to the four burial set-ups. Specifically, 111 were uniquely found in the U_A, 33 in S_A, 96 in U_An, and 40 in S_An.

Core microbiome analysis was performed at genus level using MicrobiomeAnalyst based on sample prevalence and relative abundance cut off value at $20 \%$ and $0.01 \%$, respectively (Fig. 9). In unsterilized soil-aerobic condition (U_A), 12 core bacterial genera were identified as Bacillus, Clostridium, Lactobacillus, Paraclostridium, Escherichia, Tissierella, Enterococcus, Anaerosalibacter, Rummeliibacillus, Proteus, Pediococcus, and Urmitella in the descending order according to their prevalence in samples (Fig. 9a). In contrast, 13 core bacterial genera were identified in sterilized soil-aerobic condition (S_A) in the descending order of prevalence in samples as Bacillus, Clostridium, Lactobacillus, Escherichia, Rummeliibacillus, Enterococcus, Corynebacterium, Pediococcus, Peptostreptococcus, Anaerosalibacter, Unclassified Genus Tissierellaceae, Eubacterium, and Tissierella (Fig. 9b). Bacillus was found to be the most dominant genus in U_A and S_A groups. Meanwhile, in unsterilized soil-anaerobic condition (U_An), 10 core bacterial genera were identified as Clostridium, Bacillus, Lactobacillus, Paraclostridium, Escherichia, Enterococcus, Pediococcus, Rummeliibacillus, Sporanaeobacter and Eubacterium in the descending order according to their prevalence in samples (Fig. 9c). In contrast, 11 core bacterial genera were identified in sterilized soil-anaerobic condition in the descending order of prevalence in samples as Clostridium, Bacillus, Lactobacillus, Escherichia, Enterococcus, Rummeliibacillus, Pediococcus, Virgibacillus, Eubacterium, Unclassified Genus Tissierellaceae, and Anaerosalibacter (Fig. 9d). Clostridium was the most dominant genus in U_An and S_An group. The overall core bacterial genera in all carcass decomposition group consisted of 12 genera with Bacillus as the most dominant genus among all the samples (Fig. 9e).

\section{Discussion}

\section{Swine carcass decomposition}

In the present study, the moisture content at 60 days of decomposition was observed to be high in the unsterilized soil-anaerobic burial set-up. This indicates that microbes present in the soil and carcass may have contributed to the high moisture during decomposition. This result is similar to previous studies which suggest that decomposition is affected by moisture. In terms of the effect of soil pH on the decomposition process, several research papers have suggested that bacterial growth is enhanced in neutral or slightly alkaline conditions, whereas acidic conditions promote fungal growth [10]. The pH 
values obtained in our study ranged from 6.50 to 8.16 . The $\mathrm{pH}$ progressively increased with increasing time of decomposition and remained relatively constant between days 30 to 60 . The increase in soil pH is attributed to the release of nitrogen mineralization by-products from the degradation of macromolecules [11]. Although the $\mathrm{pH}$ of the burial soil was highly variable, there were significant differences in soil $\mathrm{pH}$ between the set-ups at days 30 and 60. In the study by Ki et al. (2018) [3], the pH at the beginning of decomposition was 6.9, then decreased at day 54 before increasing up to 7.9. Several decomposition studies have provided contradictory results on the variation of soil pH over time[4]. A long-held belief is that cadaver decomposition results in increased $\mathrm{pH}$ values because of the accumulation of $\mathrm{NH}_{4}{ }^{+}$in the soil [11]. This indicates that the high pH of the samples could be a result of the ammonification of proteins and organic nitrogen [11].

\section{Bacterial diversity during carcass decomposition}

The bacterial communities are essential for maintenance of soil quality because of their involvement in organic matter dynamics, nutrient cycling and decomposition [8]. However, metabolites produced by the microorganism can affect the balance of the ecosystem [9]. Several studies were also conducted to investigate bacterial communities from leachates in animal carcass disposal sites [12] and groundwater around carcass disposal sites [13]. These studies provided vital information on the bacterial populations, as well as the bacteria which can adversely affect the environment and human health. Thus, an investigation of bacterial community composition in soil burial is important, because compounds from decomposing carcasses could affect the land and surrounding environment, and may pose human health risk.

In this study, the bacterial community analysis of animal carcass disposal soil was investigated using Illumina Miseq sequencing and the bacterial populations over the course of the decomposition period were determined. Firmicutes was found to be the dominant phylum in all samples. According to Li et al. (2013) [14], Firmicutes reduce large macromolecules such as proteins, complex fats and polycarbohydrates to their building blocks. Furthermore, Firmicutes are more prominent during active decomposition $[15,16]$.

On the other hand, phylum Proteobacteria was also abundant during days 5 and 10 of decomposition. However, there was a reduction in population after 10 days of decomposition. The phylum Proteobacteria includes a-Proteobacteria, $\beta$-Proteobacteria, $\gamma$-Proteobacteria, and $\delta$-Proteobacteria. Of these, $\gamma^{-}$ Proteobacteria are common in soil and plays an important role in the decomposition of fats and carbohydrates [17].

Bacillus, Clostridium, Lactobacillus, Escherichia, and Enterococcus were among the common genera found at days 5 and 10 of decomposition, indicating the presence of potentially pathogenic microbes in soil samples during carcass decomposition. Meanwhile, Lactobacillus spp. decreased in abundance after 10 days of decomposition. 
The abundance of Clostridium spp. is high at days 5 to 30 of incubation in the unsterilized soil both incubated aerobic and anaerobic conditions. However, abundance of Clostridium spp. under anaerobic conditions is relatively higher than in anaerobic conditions. Several studies reported that the anaerobic layer that develops around a carcass during decomposition is associated for the increase in abundance of Clostridium spp. which plays an important role in the biomass digestion. It produces a wide variety of extracellular enzymes which helps in the degradation of large biological molecules [18-20]. At the end of the decomposition period, the predominant genus in all burial set-ups was Bacillus, in which a number of species are capable of denitrification [21]. Microbial analysis suggest that anaerobic bacteria flourished at most times during decomposition. Meanwhile, aerobic bacteria flourish during the early stages of the decomposition process as there is oxygen present within the body. However, as the microbial population increases, the accumulation of gases during the decomposition process makes the environment anaerobic which prompts the microbial community to shift to anaerobic bacteria [22]. Additionally, the changes in bacterial communities suggest that various bacterial species played a role during the decomposition period.

\section{Conclusions}

In this study, we evaluated the changes in the bacterial communities in decomposing swine carcasses over 60 days period. Different bacterial genera dominated at specific period during decomposition. The composition of the bacterial community during carcass decomposition was affected by the presence or absence of soil microorganisms and the oxygen access. The dominant phylum in all samples throughout the experimental period was Firmicutes. The genera Bacillus was dominant in the burial set-ups starting from day 30 of decomposition. Overall, our results indicate that bacterial communities in the burial set-up changes continuously throughout the decomposition process of carcass.

\section{Methods}

\section{Preparation of carcass samples}

A domestic pig (Sus scrofa), weighing $10.0 \pm 2.0 \mathrm{~kg}$ was purchased commercially. All the experimental protocol in this study was approved by the Animal Care and Use Committee (Approval number: SCNU IACUC-2019-7) of Sunchon National University (Suncheon, Jeollanam-do, Korea), and performed in accordance with the guidelines and regulation set by this governing body. All methods in this experiment was carried out in compliance with the Animal Research Reporting In Vivo Experiments (ARRIVE) guidelines. The swine was euthanized and its carcass was separated from the bones, while the blood and internal organs were taken. After removing the bone, the carcass, skin, blood, and internal organs were homogenized in a blender and used for the microcosm burial set-up.

\section{Soil preparation}


To simulate conditions similar to the burial of deceased animals on a farm, soil samples were obtained from the experimental farm of Sunchon National University. Soil samples were sieved (mesh size: $2 \mathrm{~mm}$ ) and divided into two set-ups: (1) soil with an intact microbial community (unsterilized) and (2) soil that was sterilized. Soil sample was sterilized by autoclaving at $121^{\circ} \mathrm{C}, 15 \mathrm{psi}$ thrice in four days to destroy microbes, fungi and their spores [1].

\section{In vitro set-up for carcass decomposition}

A $2 \times 2$ carcass burial set-up in a laboratory setting was made using two types of soil (i. soil with an intact microbial community; ii. sterilized soil) under two incubation conditions (a. with oxygen access; $b$. without oxygen access). The treatment set-ups were as follows: unsterilized soil - aerobic condition (U_A), sterilized soil - aerobic condition (S_A), unsterilized soil - anaerobic condition (U_An), and sterilized soil anaerobic condition (S_An). The microcosm burial set-up used was based on the study conducted by Han et al [23] with some modifications. Sixty sterilized containers $(450 \mathrm{~mL})$ containing $120 \mathrm{~g}$ of soil and $45 \mathrm{~g}$ of meat were placed in each container. The soil/carcass ratio were homogenized thoroughly in order to evenly distribute the meat within the soil. The mixture of soil and meat was decomposed under aerobic and anaerobic conditions. Anaerobic conditions were created by sealing all parts of the container and placed in an incubator with $5 \%$ flowing $\mathrm{CO}_{2}$. For simulating aerobic conditions, the lid of the reactor was punctured so that air could pass through the hole prior to placing it in an incubator. All experimental setups were conducted in triplicate, incubated at $25^{\circ} \mathrm{C}$ for a total period of 60 days and sampled at days: 0 , $5,10,30$, and 60 . Day 0 is the initial placement followed by subsequent sample collections on days 5,10 , 30 , and 60 . Two grams of samples were taken from each container and stored at $-20^{\circ} \mathrm{C}$ for chemical and metagenomic analyses.

\section{Chemical analysis}

Samples were collected at days $0,5,10,30$, and 60 . To measure the $\mathrm{pH}$ of the sample, $1 \mathrm{~g}$ of soil was suspended in $5 \mathrm{ml}$ sterilized distilled water and vortexed for $1 \mathrm{~min}$. The supernatant was collected after allowing the large particles to settle for $5 \mathrm{~min}$ and the $\mathrm{pH}$ was measured with a pH meter (SevenCompact ${ }^{\mathrm{TM}} \mathrm{pH} /$ Ion meter S220, Mettler Toledo, Switzerland). The average readings of the three samples was used to estimate the $\mathrm{pH}$ for each soil [1]. The moisture content of the soil and carcass mixture was estimated according to the standard method AS 1289 B1.1. One gram of burial soil was weighed, placed in an aluminum plate, and oven-dried overnight at $105^{\circ} \mathrm{C}$.

\section{DNA extraction, PCR amplification and sequencing}

Metagenomic DNA was extracted from $0.25 \mathrm{~g}$ of soil samples obtained from each burial set-up were using the DNeasy ${ }^{\circledR}$ PowerSoil ${ }^{\circledR}$ Kit (Qiagen, Hilden, Germany) according to the manufacturer's instructions. The extracted DNA was stored at $-20^{\circ} \mathrm{C}$ until further processing. The quality and quantity of the extracted DNA was checked using Quant-IT PicoGreen (Invitrogen, Grand Island, NY, USA). DNA sequencing libraries targeting the V3-V4 hypervariable region of the 16S rRNA gene were performed according to the Illumina $16 \mathrm{~S}$ metagenomic sequencing library preparation method [24]. This consists of 
two PCR steps. In the first amplification, specific primers were used, while in the second, index information for sample identification was added. The DNA was amplified by primary PCR using universal primer pair with Illumina adapter overhang sequences, S-D-Bact-0341-b-S-1 (5'-TCG TCG GCA GCG TCA GAT GTG TAT AAG AGA CAG CCT ACG GGN GGC WGC A-3') and S-D-Bact-0785-a-A-21 (5'-GTC TCG TGG GCT CGG AGA TGT GTA TAA GAG ACA GGA CTA CHV GGG TAT CTA ATC C-3') [25]. The PCR was performed with $2.5 \mu \mathrm{L}$ of DNA sample $(5 \mathrm{ng} / \mu \mathrm{L}), 5 \mu \mathrm{L}$ each of the universal forward and reverse primer, and $12.5 \mu \mathrm{L}$ of $2 x$ KAPA HiFi HotStart ReadyMix (KAPA Biosystems, Wilmington, MA, USA) in a total volume of $25 \mu \mathrm{L}$. The cycle condition comprised an initial denaturation at $95^{\circ} \mathrm{C}$ for $3 \mathrm{~min}$, followed by 25 cycles of denaturation at $95^{\circ} \mathrm{C}$ for $30 \mathrm{~s}$, annealing at $55^{\circ} \mathrm{C}$ for $30 \mathrm{~s}$, and extension at $72{ }^{\circ} \mathrm{C}$ for $30 \mathrm{~s}$, and a final extension at $72{ }^{\circ} \mathrm{C}$ for $5 \mathrm{~min}$. The PCR products were purified with AMPure XP beads (Agencourt Bioscience, Beverly, MA) to remove free primers and primer dimer species. Following purification, $2 \mathrm{uL}$ of the primary PCR product was amplified for secondary PCR for library construction. Primer sequences used for the secondary PCR are as follows: a Nextera XT Index primer pair (Illumina ${ }^{\circledR}$, USA), Primer 1 (N7xx): 5' - AAT GAT ACG GCG ACC ACC GAG ATC TAC AC - [i5] - TCG TCG GCA GCG TC -3' and Primer 2 (S5xx): 5' - CAA GCA GAA GAC GGC ATA CGA GAT - [i7] - GTC TCG TGG GCT CGG - 3'. The PCR consisted of $5 \mu \mathrm{L}$ of sample DNA, $5 \mu \mathrm{L}$ each of Nextera XT Index primers 1 and 2, $25 \mu \mathrm{L}$ of $2 x \mathrm{KAPA} \mathrm{HiFi}$ HotStart ReadyMix (KAPA Biosystems, Wilmington, MA, USA), and $10 \mu \mathrm{L}$ of PCR Grade Water. The cycle conditions comprised an initial denaturation at $95{ }^{\circ} \mathrm{C}$ for $3 \mathrm{~min}$, followed by 8 cycles of denaturation at 95 ${ }^{\circ} \mathrm{C}$ for $30 \mathrm{~s}$, annealing at $55^{\circ} \mathrm{C}$ for $30 \mathrm{~s}$, and extension at $72{ }^{\circ} \mathrm{C}$ for $30 \mathrm{~s}$, and a final extension at $72{ }^{\circ} \mathrm{C}$ for $5 \mathrm{~min}$. The final PCR product (final library) was cleaned-up before quantification using the AMPure XP beads (Agencourt Bioscience, Beverly, MA). Finally, the PCR products were quantified using qPCR according to the qPCR Quantification Protocol Guide (KAPA Library Quantification kit for Illumina Sequencing platforms) and library quality was assessed using the TapeStation D1000 ScreenTape (Agilent Technologies, Waldbronn, Germany). Equimolar amounts of the barcoded V3-V4 amplicons were pooled and paired-end sequenced $(2 \times 300 \mathrm{bp})$ on an Illumina MiSeq ${ }^{\circledR}$ platform (Illumina Inc., San Diego, California, USA) using v3 reagents, according to the manufacturer's instructions at the Macrogen Inc. (Seoul, South Korea).

\section{Sequence data processing and analysis}

The raw data files (fastq) containing the sequenced paired-end reads were obtained using the bcls2fastq package (Illumina Inc., San Diego, CA, USA) from the base call binary data produced by real-time analysis. Raw sequences were pre-processed to filter the adaptor sequences and remove low-quality sequences using the Trimmomatic v0.38 [26] and assembled using Fast Length Adjustment of Short Reads (FLASH 1.2.11) [27]. Sequences shorter than 400 bp were discarded to ensure that any subsequent analysis was highly accurate. The obtained sequences were subjected to CD-HIT-OTU [28] to remove lowquality sequences, ambiguous sequences, and chimera sequences, which are considered as sequencing errors. The filtered reads were clustered and identified as OTU at $97 \%$ sequence similarity using CD-HITOTU [28], and chimeric sequences were identified and removed using rDnaTools (https://github.com/PacificBiosciences/rDnaTool). The representative sequences from the clustered OTU were taxonomically assigned using Quantitative Insights Into Microbial Ecology (QIIME Version 1) [29] 
from the NCBI 16S rRNA database, and the taxonomy composition from phylum to species level was generated using QIIME-UCLUST [30].

The metagenomic data was visualized using a biom formatted OTU table [31] in the MicrobiomeAnalyst tool (http://www.microbiomeanalyst.ca) [32]. The OTU data were filtered in order to remove low quality or uninformative feature. The following criteria were used: low count filter was set using default minimum count of 4 and the prevalence in samples was set at $20 \%$, while the low variance filter was set at interquartile range and the percentage to remove was set at $10 \%$. The data were normalized by rarefying to the minimum library size and scaled by total sum scaling before further downstream processing.

Alpha diversity of each sample was assessed using the observed OTU number and Chao1 to measure the species richness, whereas species evenness was measured using Shannon index. Significant differences between samples in alpha-diversity were assessed using a non-parametric Kruskal-Wallis test. Beta diversity was calculated based on Bray-Curtis dissimilarity and statistical significance of the clustering pattern in the ordination plot was evaluated by permutational multivariate analysis of variance (PERMANOVA). The ordination plot was visualized using non-metric multidimensional scaling (NMDS) graph. The core microbiome analysis was done as described in MicrobiomeAnalyst [32]. To detect the core microbiome, $20 \%$ prevalence and $0.01 \%$ relative abundance was used. The relative abundances of the phylum, genus, and species levels were plotted as a bar graph. Hierarchical cluster analysis was visualized using the MicrobiomeAnalyst tool using Euclidean distance measure and Ward clustering algorithm of relative abundances of bacterial OTUs at the order level. In addition, a Venn diagram of the membership-based representation of unique, shared and core bacterial community was generated in MetaCOMET [33] using jvenn [34].

\section{Statistical Analysis}

Data for moisture and $\mathrm{pH}$ were subjected to analysis of variance (ANOVA) using the general linear model (GLM) procedure of Statistical Analysis Systems (SAS) version 9.4 (SAS Institute Inc., Cary, NC, USA). All analyses were conducted in triplicate and Duncan's multiple range test was used to identify differences between specific treatments. Differences with $p$ values less than 0.05 were considered statistically significant.

\section{Declarations}

\section{Acknowledgments}

The authors gratefully acknowledge financial assistance from the Korea Institute of Planning and Evaluation for Technology in Food, Agriculture and Forestry (IPET) funded by the Ministry of Agriculture, Food and Rural Affairs (MAFRA). We also express our appreciation to all members of the Animal Disease and Diagnostic Laboratory (ADDL) and Ruminant Nutrition and Anaerobe Laboratory (RNAL) for their assistance in the conduct of the study. 


\section{Authors' contributions}

Co-conceptualized the study: All. Performed the experiments: MM. Analyzed the data: MM, SHK. Wrote the manuscript: MM. Supervision: SSL, YIC. Funding acquisition: SHK, YIC. Undertook critical revision of the manuscript: All. All authors reviewed, edited, and approved the final manuscript.

\section{Funding}

This work was supported by the Korea Institute of Planning and Evaluation for Technology in Food, Agriculture and Forestry (IPET) through the Animal Disease Management Technology Development Program, funded by the Ministry of Agriculture, Food and Rural Affairs (MAFRA) (118099-03).

\section{Availability of data and materials}

The data generated and used in this study are available from the corresponding author upon reasonable request.

\section{Ethics approval and consent to participate}

All experimental protocol of this study was approved by the Animal Care and Use Committee (Approval number: SCNU IACUC-2019-7) of Sunchon National University (Suncheon, Jeollanam-do, Korea). All experiments were performed in accordance with the guidelines and regulation set by this governing body. All methods in this study was carried out in compliance with the ARRIVE guidelines.

\section{Consent for publication}

Not applicable.

\section{Competing interests}

The authors declare that they have no competing interests.

\section{Author details}

Department of Animal Science and Technology, Sunchon National University, Suncheon, Jeonnam 57922, Republic of Korea

\section{Abbreviations}

ANOVA: Analysis of variance; FLASH: Fast Length Adjustment of Short Reads; GLM: General linear model; OTU: Operational taxonomic unit; PERMANOVA: Permutational multivariate analysis of variance; QIIME: Quantitative Insights Into Microbial Ecology; SAS: Statistical analysis systems;

\section{References}


1. Lauber CL, Metcalf JL, Keepers K, Ackermann G, Carter DO, Knight R. Vertebrate Decomposition Is Accelerated by Soil Microbes. Appl Environ Microbiol. 2014;80:4920-29.

2. Gwyther CL, Williams AP, Golyshin PN, Edwards-Jones G, Jones DL. The environmental and biosecurity characteristics of livestock carcass disposal methods: A review. Waste Management. 2011;31:767-78.

3. Ki BM, Kim YM, Jeon JM, Ryu HW, Cho KS. Characterization of odor emissions and microbial community structure during degradation of pig carcasses using the soil burial-composting method. Waste Manag. 2018;77:30-42.

4. Vass A. Beyond the grave - understanding human decomposition. Microbiol Today. 2001;28:190-2. http://www.academia.dk/BiologiskAntropologi/Tafonomi/PDF/ArpadVass_2001.pdf. Accessed 3 Nov 2020.

5. Rapp D, Potier P, Jocteur-Monrozier L, Richaume A. Prion degradation in soil: Possible role of microbial enzymes stimulated by the decomposition of buried carcasses. Environ Sci Technol. 2006;40:6324-9.

6. Valseth K, Nesbø CL, Easterday WR, Turner WC, Olsen JS, Stenseth NC, et al. Temporal dynamics in microbial soil communities at anthrax carcass sites. BMC Microbiol. 2017;17:206.

7. Howard GT, Duos B, Watson-Horzelski EJ. Characterization of the soil microbial community associated with the decomposition of a swine carcass. Int Biodeterior Biodegradation. 2010;64:3004.

8. Acosta-Martínez V, Acosta-Mercado D, Sotomayor-Ramírez D, Cruz-Rodríguez L. Microbial communities and enzymatic activities under different management in semiarid soils. Appl Soil Ecol. 2008;38:249-60.

9. Stahl D, Tiedje J. Microbial Ecology and Genomics: A Crossroads of Opportunity. 2002. https://doi:10.1128/AAMCOL.23FEB.2001.

10. Rousk J, Brookes PC, Bååth E. Contrasting Soil pH Effects on Fungal and Bacterial Growth Suggest Functional Redundancy in Carbon Mineralization. Appl Environ Microbiol. 2009;75:1589-96.

11. Hopkins D., Wiltshire PE., Turner B. Microbial characteristics of soils from graves: an investigation at the interface of soil microbiology and forensic science. Appl Soil Ecol. 2000;14:283-8.

12. Park J-A, Choi N-C, Kim S-B. Analysis of Microbial Communities in Animal Carcass Disposal Soils. J Korean Soc Environ Eng. 2013;35:503-8.

13. Koh EH, Kaown D, Kim HJ, Lee KK, Kim H, Park S. Nationwide groundwater monitoring around infectious-disease-caused livestock mortality burials in Korea: Superimposed influence of animal leachate on pre-existing anthropogenic pollution. Environ Int. 2019;129:376-88.

14. Li L, He Q, Ma Y, Wang X, Peng X. A mesophilic anaerobic digester for treating food waste: Process stability and microbial community analysis using pyrosequencing. Microb Cell Fact. 2016;15:65.

15. Cobaugh KL, Schaeffer SM, DeBruyn JM. Functional and Structural Succession of Soil Microbial Communities below Decomposing Human Cadavers. PLoS One. 2015;10:e0130201. 
16. Weiss S, Carter DO, Metcalf JL, Knight R. Carcass mass has little influence on the structure of gravesoil microbial communities. Int J Legal Med. 2016;130:253-63.

17. Sanapareddy N, Hamp TJ, Gonzalez LC, Hilger HA, Fodor AA, Clinton SM. Molecular Diversity of a North Carolina Wastewater Treatment Plant as Revealed by Pyrosequencing. Appl Environ Microbiol. 2009;75:1688-96.

18. Tracy BP, Jones SW, Fast AG, Indurthi DC, Papoutsakis ET. Clostridia: the importance of their exceptional substrate and metabolite diversity for biofuel and biorefinery applications. Curr Opin Biotechnol. 2012;23:364-81.

19. Berge ACB, Glanville TD, Millner PD, Klingborg DJ. Methods and microbial risks associated with composting of animal carcasses in the United States. J Am Vet Med Assoc. 2009;234:47-56.

20. Yang SH, Ahn HK, Kim BS, Chang SS, Chung KY, Lee EM, et al. Comparison of bacterial communities in leachate from decomposing bovine carcasses. Asian-Australasian J Anim Sci. 2017;30:1660-6. doi:10.5713/ajas.17.0553.

21. Sun Y, De Vos P, Heylen K. Nitrous oxide emission by the non-denitrifying, nitrate ammonifier Bacillus licheniformis. BMC Genomics. 2016;17.

22. Janaway RC. The decomposition of materials associated with buried cadavers. 2008. https://bradscholars.brad.ac.uk/handle/10454/4713. Accessed 2 Nov 2020.

23. Han I, Yoo K, Kang BR, No JH, Wee GN, Khan MI, et al. A comparison study of the potential risks induced in arable land and forest soils by carcass-derived pollutants. Environ Geochem Health. 2018;40:451-60.

24. Illumina Inc. 16 S Metagenomic Sequencing Library Preparation - Preparing 16 S Ribosomal RNA Gene Amplicons for the Illumina MiSeq System. 16S Metagenomic Sequencing Library Preparation Manual. 2013.

https://support.illumina.com/documents/documentation/chemistry_documentation/16s/16smetagenomic-library-prep-guide-15044223-b.pdf. Accessed 25 Oct 2020.

25. Klindworth A, Pruesse E, Schweer T, Peplies J, Quast C, Horn M, et al. Evaluation of general 16S ribosomal RNA gene PCR primers for classical and next-generation sequencing-based diversity studies. Nucleic Acids Res. 2013;41:e1-e1.

26. Bolger AM, Lohse M, Usadel B. Trimmomatic: a flexible trimmer for Illumina sequence data. Bioinformatics. 2014;30:2114-20.

27. Magoc T, Salzberg SL. FLASH: fast length adjustment of short reads to improve genome assemblies. Bioinformatics. 2011;27:2957-63.

28. Li W, Fu L, Niu B, Wu S, Wooley J. Ultrafast clustering algorithms for metagenomic sequence analysis. Brief Bioinform. 2012;13:656-68.

29. Caporaso JG, Kuczynski J, Stombaugh J, Bittinger K, Bushman FD, Costello EK, et al. QIIME allows analysis of high-throughput community sequencing data. Nat Methods. 2010;7:335-6.

30. Edgar RC. Search and clustering orders of magnitude faster than BLAST. Bioinformatics. 2010;26:2460-1. 
31. McDonald D, Clemente JC, Kuczynski J, Rideout JR, Stombaugh J, Wendel D, et al. The Biological Observation Matrix (BIOM) format or: how I learned to stop worrying and love the ome-ome. Gigascience. 2012;1:7.

32. Dhariwal A, Chong J, Habib S, King IL, Agellon LB, Xia J. MicrobiomeAnalyst: a web-based tool for comprehensive statistical, visual and meta-analysis of microbiome data. Nucleic Acids Res. 2017;45:W180-8.

33. Wang Y, Xu L, Gu YQ, Coleman-Derr D. MetaCoMET: a web platform for discovery and visualization of the core microbiome. Bioinformatics. 2016;32:3469-3470.

34. Bardou P, Mariette J, Escudié F, Djemiel C, Klopp C. jvenn: an interactive Venn diagram viewer. BMC Bioinformatics. 2014;15:293.

\section{Figures}

(a)

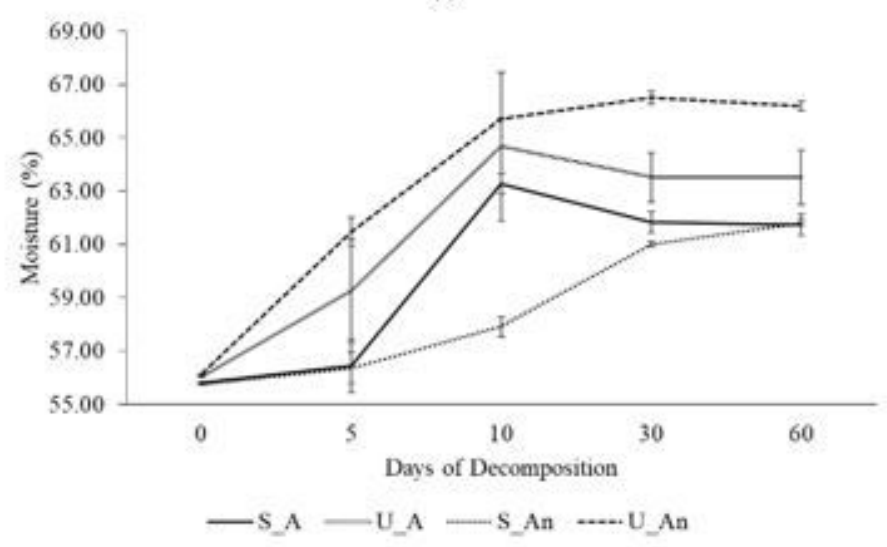

(b)

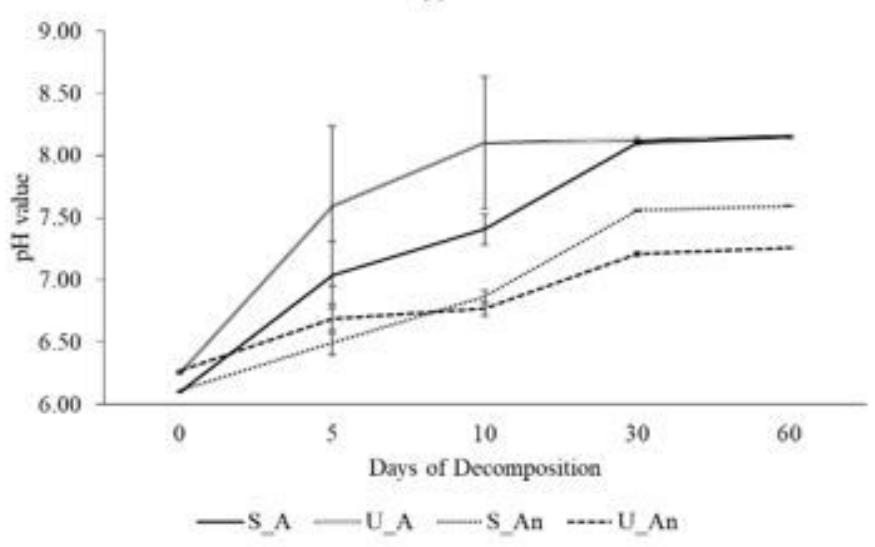

\section{Figure 1}

Chemical composition during swine carcass decomposition. (a) Moisture content and (b) pH of decomposing swine carcass in unsterilized and sterilized soil under aerobic and anaerobic condition over 60 days. (Note: $U$ and S: unsterilized and sterilized soil, respectively; $A$ and An: aerobic and anaerobic condition, respectively). 
(a)

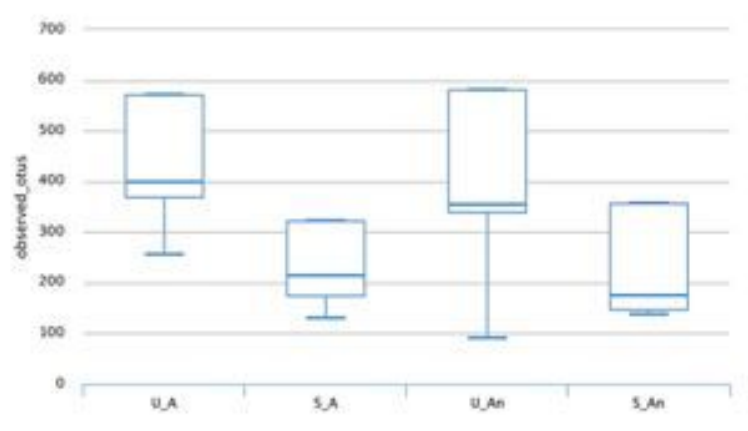

(b)

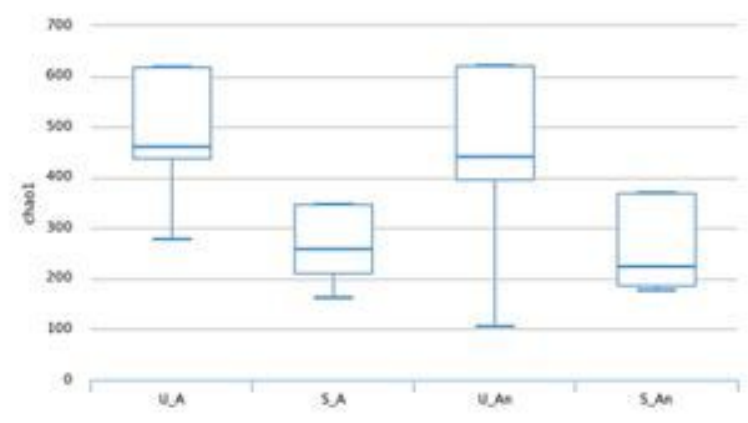

(c)

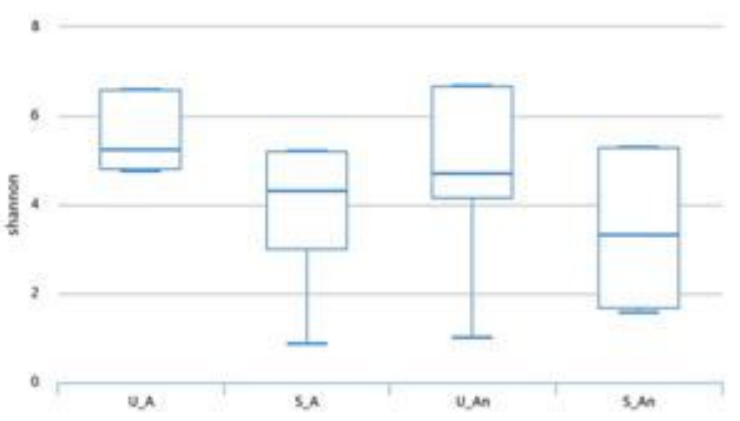

\section{Figure 2}

Box-plot representation of alpha diversity indices between groups during carcass decomposition: (a) observed OTUs, (b) Chao1, and (c) Shannon index. U_A (unsterilized soil - aerobic condition), S_A (sterilized soil - aerobic condition), U_An (unsterilized soil - anaerobic condition), and S_An (sterilized soil anaerobic condition) 
(a)

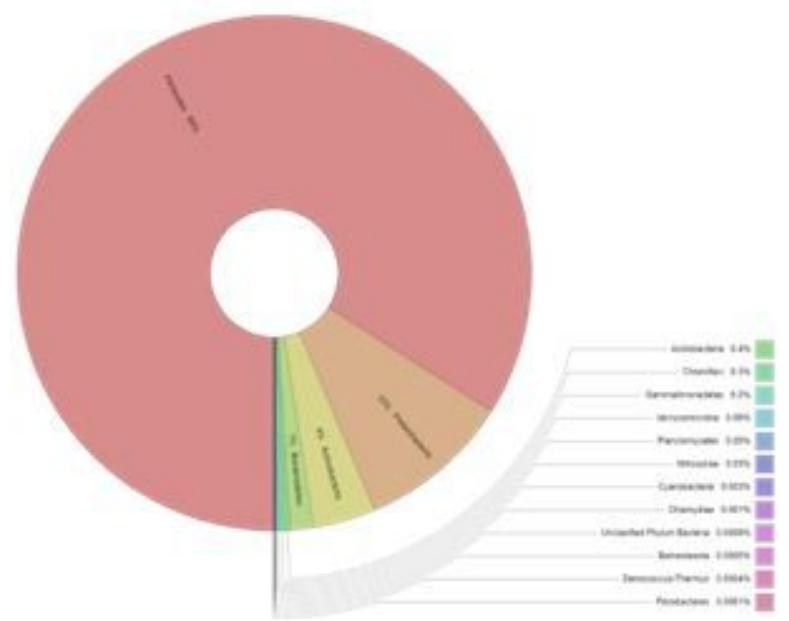

(b)

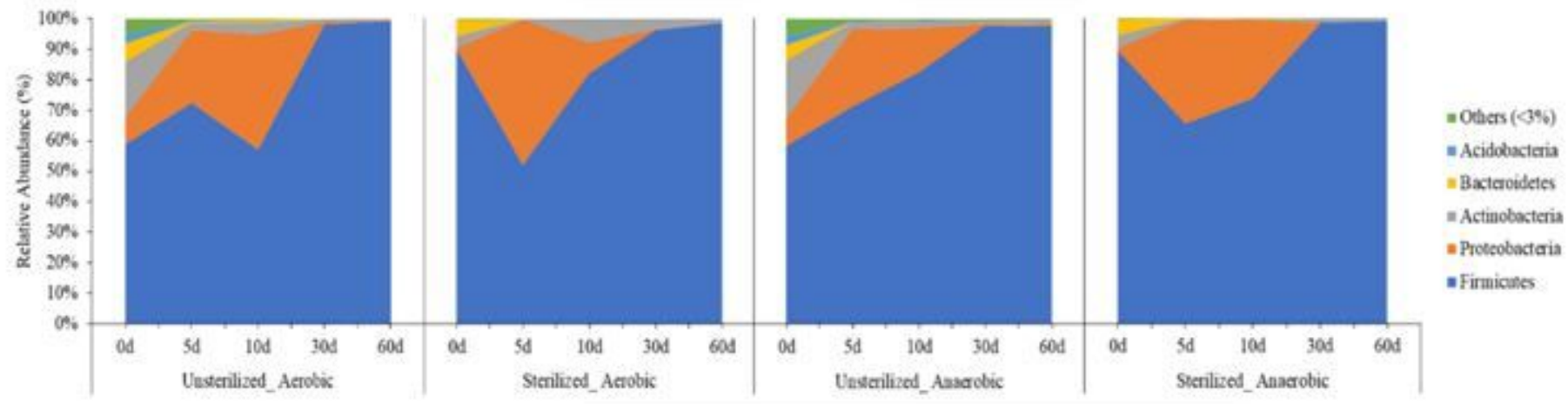

Figure 3

Relative abundance at phylum level during carcass decomposition. (a) Over all bacterial community at phylum level. (b) Bacterial community structure at the phylum level during carcass decomposition at different burial set-ups and time point.

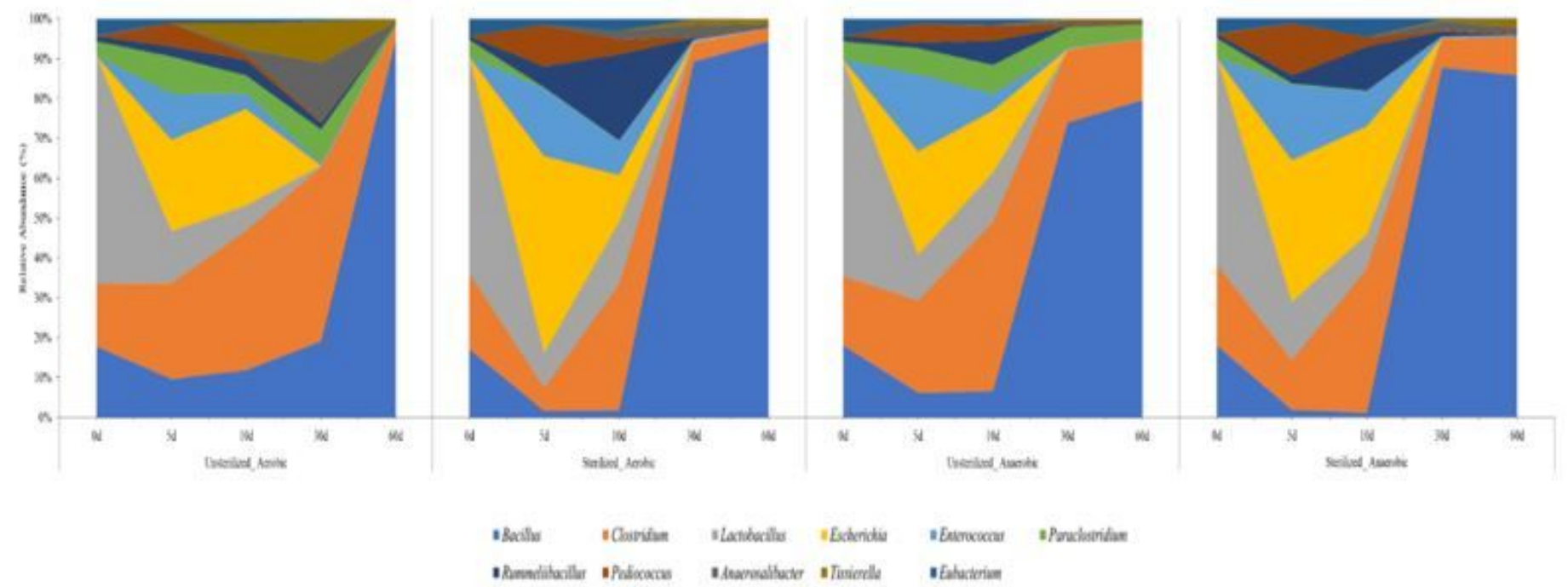


Figure 4

Bacterial community structure at the genus level during carcass decomposition.

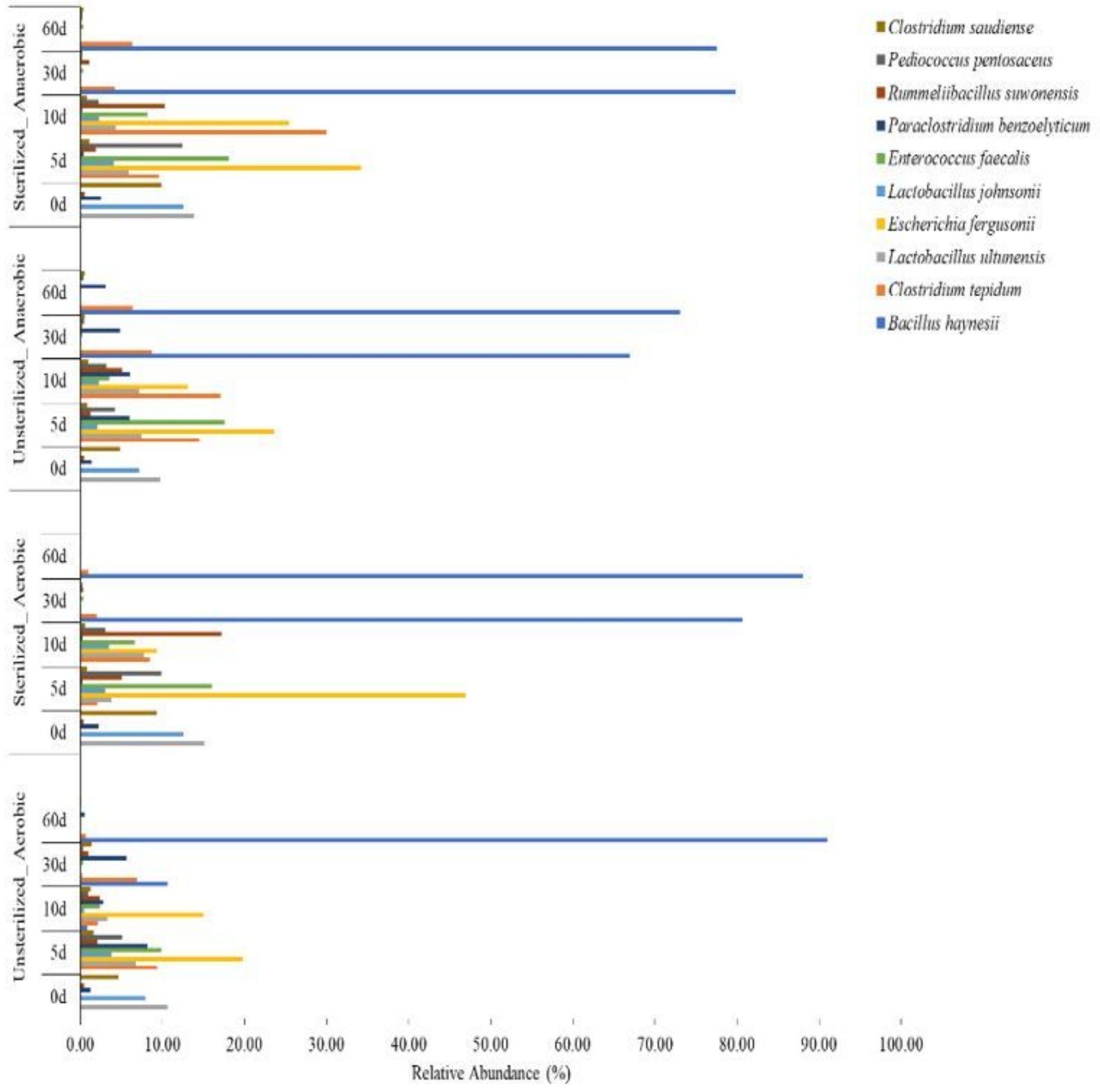

Figure 5

Bacterial community structure at the species level during carcass decomposition. 


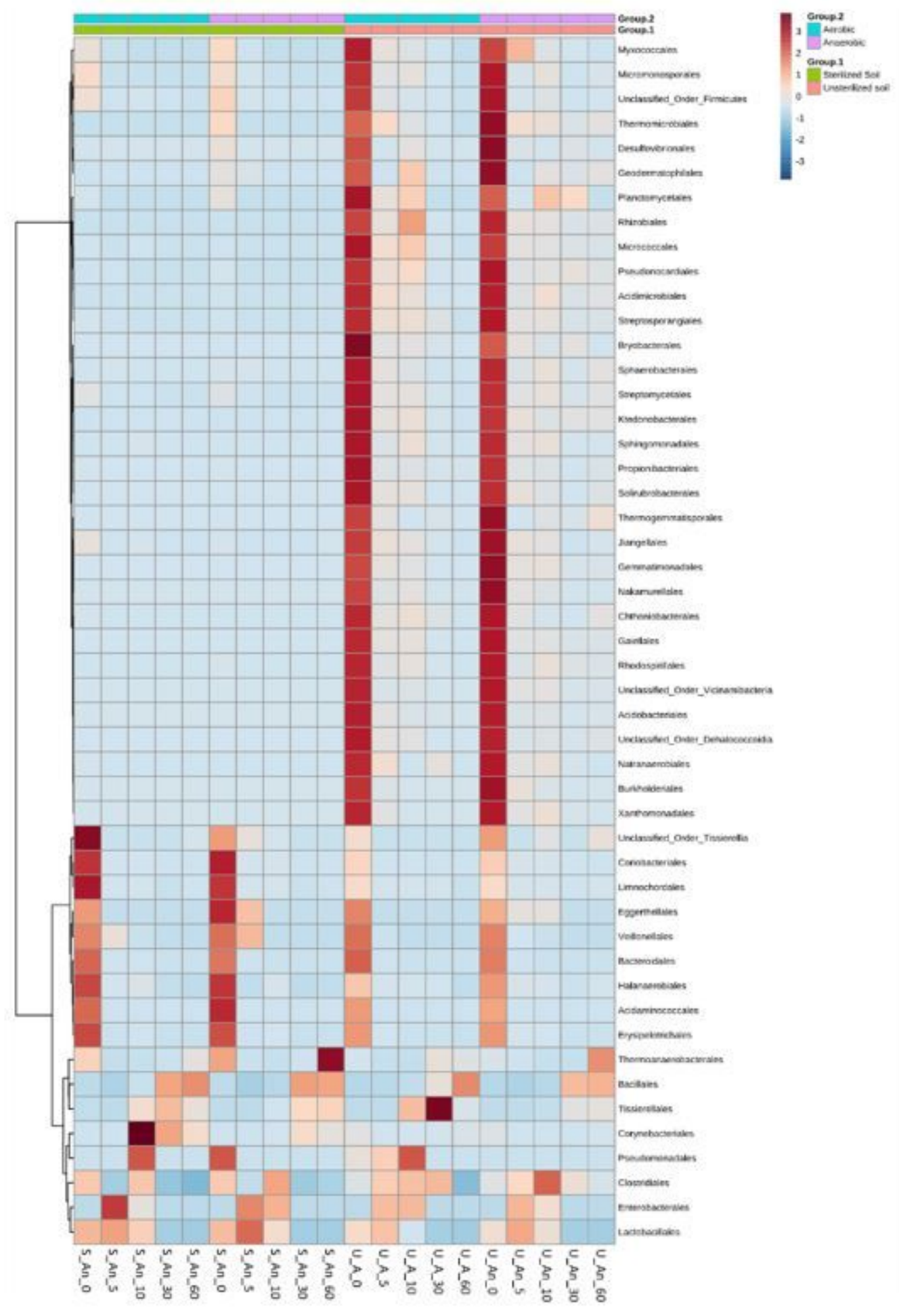

\section{Figure 6}

Hierarchical clustering heatmap of bacterial orders from the swine carcass decomposition set-up generated by MicrobiomeAnalyst using Euclidean distance measure and Ward clustering algorithm. Normalized relative abundance are plotted from low (blue), mid (peach), and high (red). U_A (unsterilized soil - aerobic condition), S_A (sterilized soil - aerobic condition), U_An (unsterilized soil - anaerobic 
condition), and S_An (sterilized soil - anaerobic condition). The $0,5,10,30$, and 60 represents sampling period.

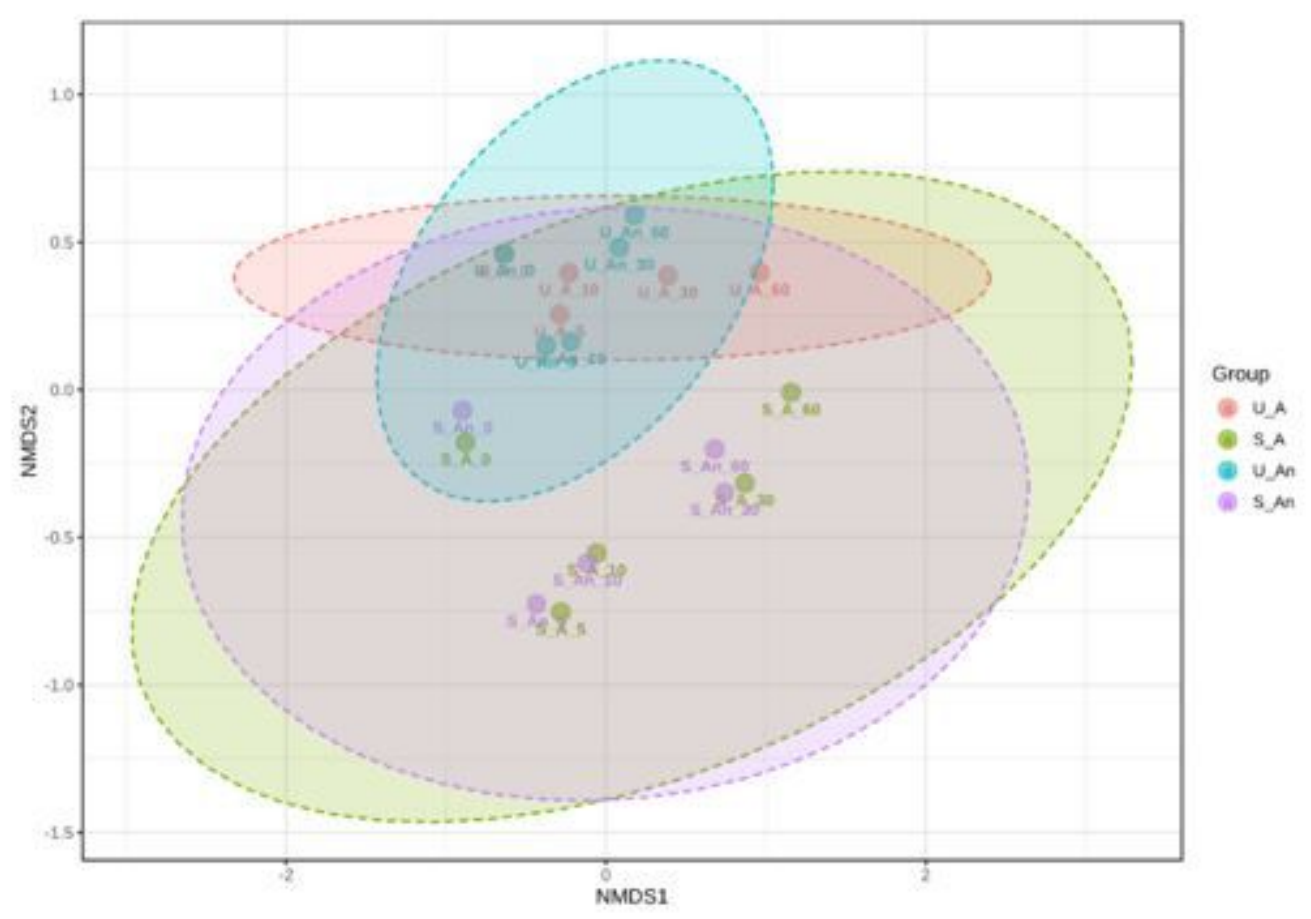

\section{Figure 7}

Non-metric multidimensional scaling (NMDS) plot based on Bray-Curtis dissimilarities of samples based on the burial groups. U_A (unsterilized soil - aerobic condition), S_A (sterilized soil - aerobic condition), U_An (unsterilized soil - anaerobic condition), and S_An (sterilized soil - anaerobic condition). The $0,5,10$, 30 , and 60 represents sampling period. 


\section{3}

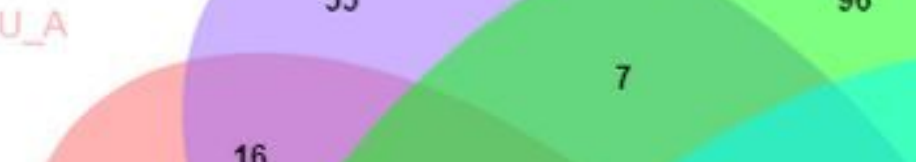

16
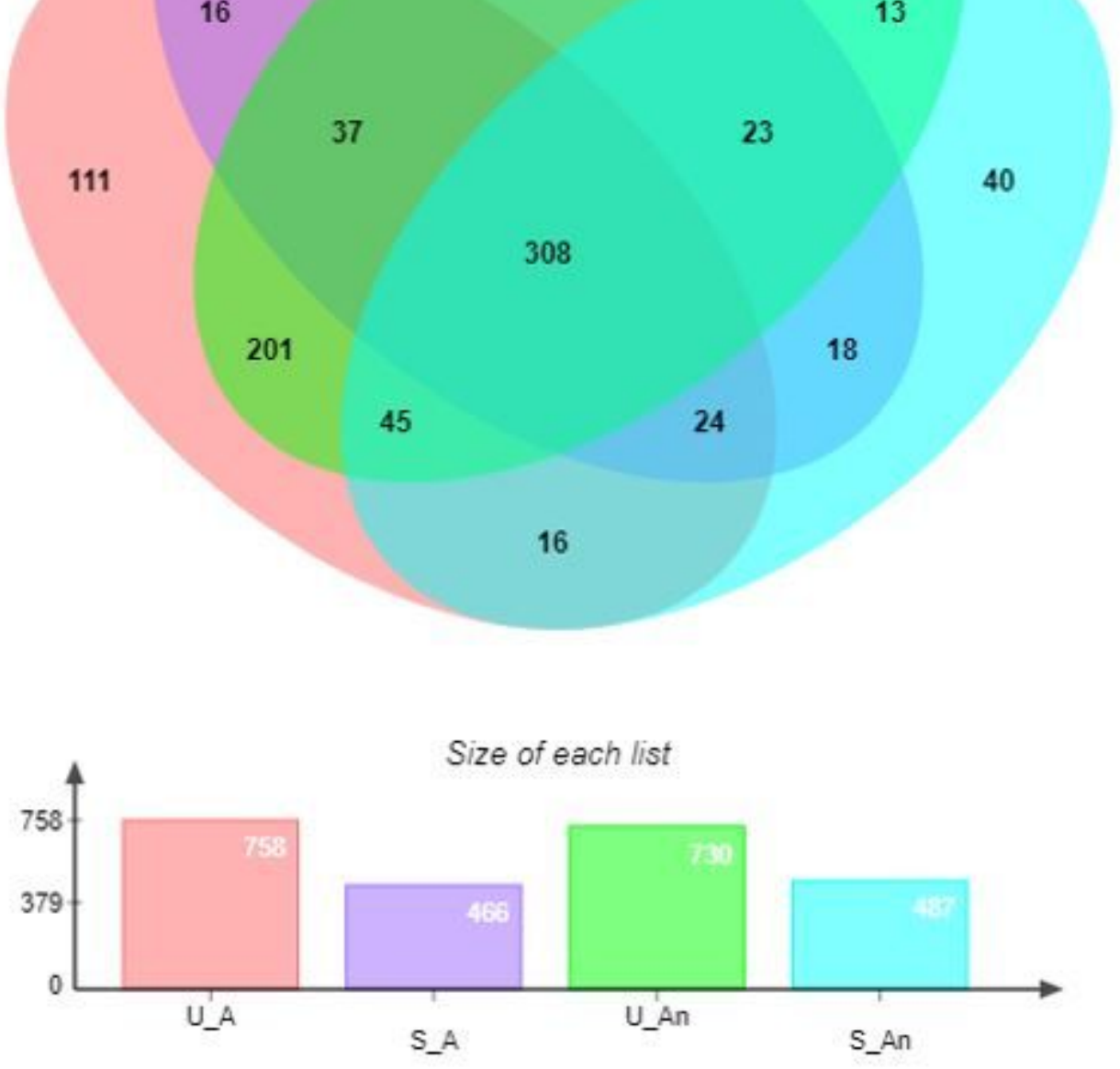

Number of elements: specific (1) or shared by 2,3,... lists

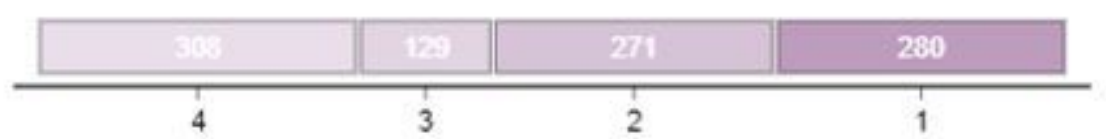

\section{Figure 8}

Membership-based representation of unique, shared and core bacterial community of decomposing swine carcass under different burial set-up conditions, and the total size of observed species per set-up. U_A (unsterilized soil, aerobic condition), S_A (sterilized soil, aerobic condition), U_An (unsterilized soil, anaerobic condition), and S_An (sterilized soil, anaerobic condition). 
(a)

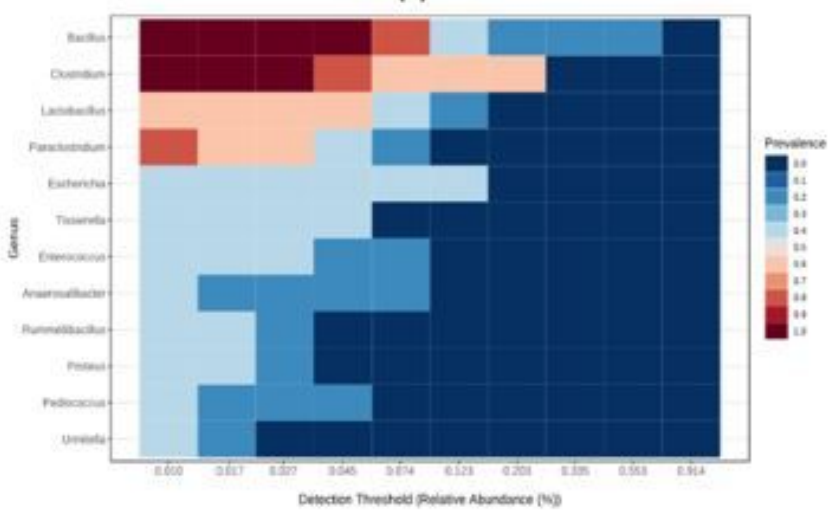

(c)

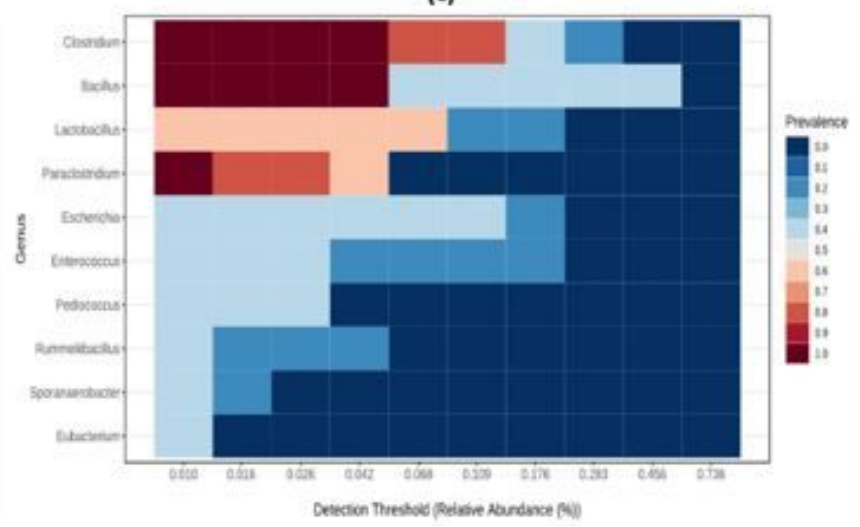

(b)

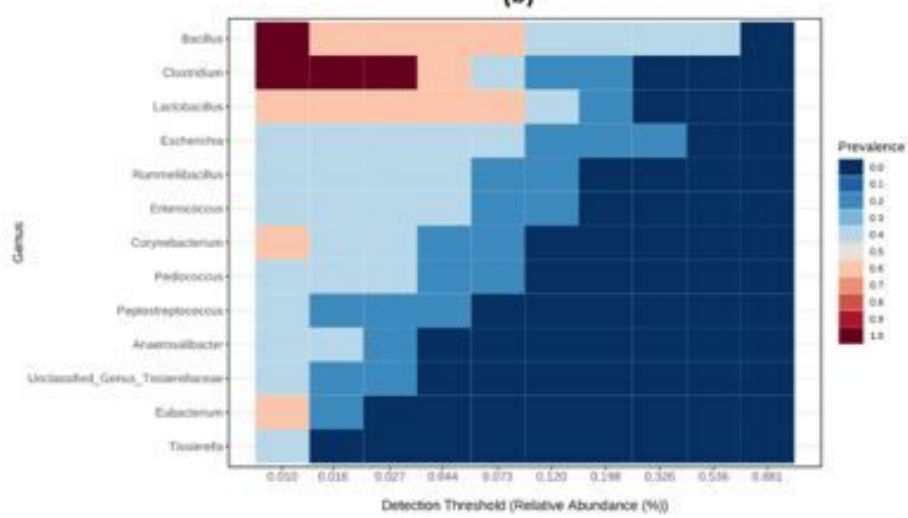

(d)

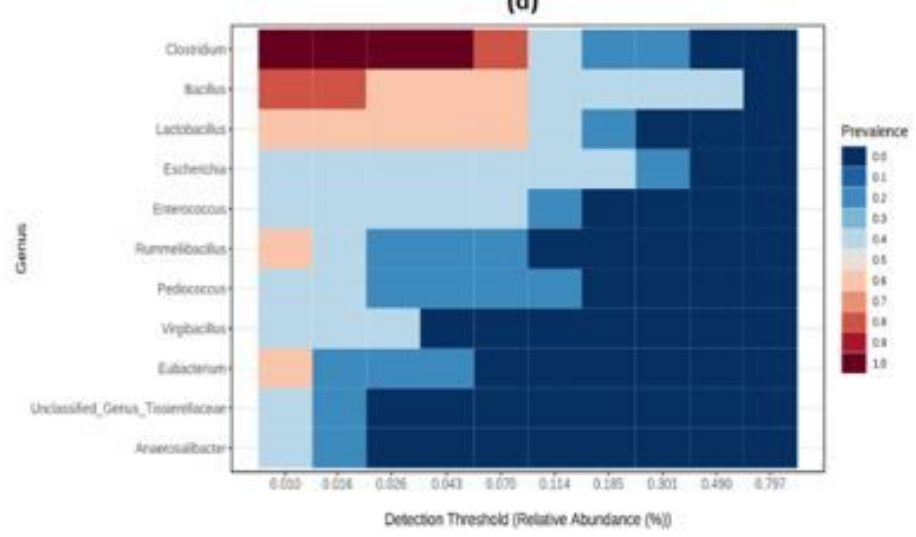

(e)

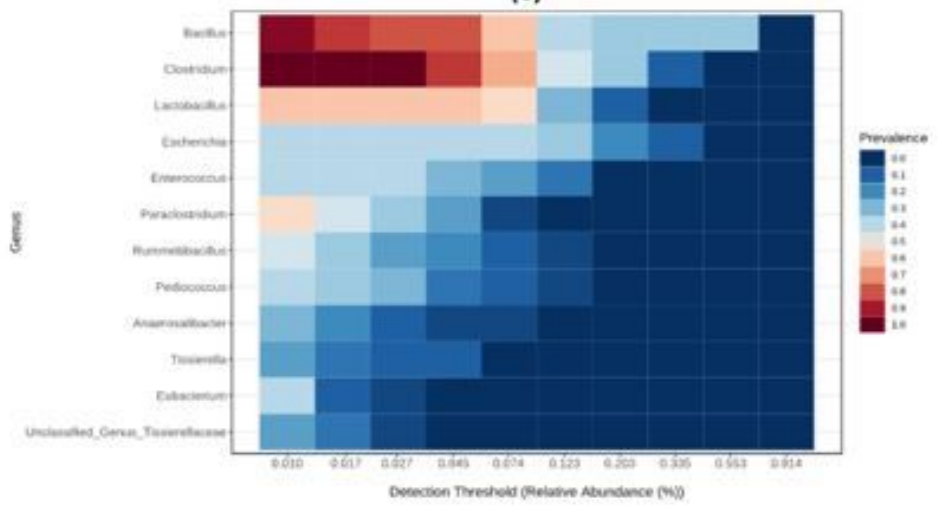

\section{Figure 9}

Heatmap representing the core microbiome at Genus level. Core microbiome at (a) unsterilized soilaerobic condition (U_A), (b) sterilized soil-aerobic condition (S_A), (c) unsterilized soil-anaerobic condition (U_An), (d) sterilized soil-anaerobic condition (S_An), and (e) the shared microbiome among the burial types. 